Cahiers $d u$ MONDE RUSSE

\section{Cahiers du monde russe}

Russie - Empire russe - Union soviétique et États indépendants

$47 / 4 \mid 2006$

Varia

\title{
Alessandra Tosi, Waiting for Pushkin
}

Rodolphe Baudin

\section{OpenEdition \\ Journals}

Édition électronique

URL : https://journals.openedition.org/monderusse/6695

DOI : 10.4000/monderusse. 6695

ISSN : $1777-5388$

Éditeur

Éditions de l'EHESS

Édition imprimée

Date de publication : 30 décembre 2006

Pagination : 800-803

ISBN : 978-2-7132-2098-2

ISSN : $1252-6576$

Référence électronique

Rodolphe Baudin, «Alessandra Tosi, Waiting for Pushkin », Cahiers du monde russe [En ligne], 47/4 I

2006, mis en ligne le 03 juillet 2009, consulté le 03 septembre 2022. URL : http://

journals.openedition.org/monderusse/6695; DOI : https://doi.org/10.4000/monderusse.6695

Ce document a été généré automatiquement le 3 septembre 2022

Tous droits réservés 


\title{
Alessandra Tosi, Waiting for Pushkin
}

\author{
Rodolphe Baudin
}

\section{RÉFÉRENCE}

Alessandra TOSI, Waiting for Pushkin. Russian Fiction in the Reign of Alexander I

(1801-1825). Amsterdam-New York : Rodopi, 2006, 429 p. (Studies in Slavic Literatureand Poetics, 15)

1 Reléguée au second plan de l'histoire littéraire par les études sur le développement de la poésie préromantique et romantique en Russie, la prose de fiction de l'époque d'Alexandre I ${ }^{\mathrm{er}}$ reste largement ignorée. De rares études existent bien depuis quelques années, mais elles se concentrent majoritairement sur un aspect spécifique de la prose des années 1800-1810, tel le roman gothique par exemple, sans présenter un panorama complet de la production de l'époque. C'est donc une lacune de la bibliographie que l'ouvrage d'Alessandra Tosi vient aujourd'hui très heureusement combler.

Organisé en cinq parties, Russian Fiction in the Reign of Alexander I s'intéresse d'abord au contexte social et culturel du début $\mathrm{du} \mathrm{XIX}^{\mathrm{e}}$ siècle. L'auteur y montre comment le libéralisme du jeune Alexandre profita au développement de la littérature dans les années 1800 . Dans cette première partie est également rappelé l'impact de la campagne de 1812-1814 sur la culture russe, qui rapprocha la Russie de l'Europe, en atténuant son complexe d'infériorité vis-à-vis de l'Occident. L'époque est également le théâtre d'un développement rapide de l'édition, en même temps que s'affirme socialement l'image de l'écrivain, dont l'activité, valorisée par la culture des salons, gagne en respectabilité. Ce progrès, comme le note $\mathrm{A}$. Tosi, a toutefois un prix : la limitation de la liberté de création imposée par le respect de la culture mondaine qui promeut la littérature. Parce qu'ils cherchent au cours du règne à s'émanciper de cette tutelle, les écrivains vont déplacer le milieu littéraire de l'espace des salons vers celui des cercles littéraires, nombreux à l'époque d'Alexandre, et qui sont au centre de la deuxième partie de l'ouvrage. 
3 Favorisant la professionnalisation du métier d'écrivain, ces sociétés stimulèrent le développement de la critique littéraire. Dans le domaine de la prose, les conceptions esthétiques du sentimentalisme imposées par Karamzin furent ainsi battues en brèche par une jeune génération de critiques comme Aleksandr Benickij ou Nikolaj Brusilov. À l'heure où la victoire de 1812 stimulait le patriotisme russe, la critique reprochait à Karamzin son allégeance au modèle linguistique français et son attention exclusive à l'intime, au détriment de la problématique nationale. Enfin, certains critiques condamnaient les excès des épigones de Karamzin, dont les œuvres outraient les caractéristiques $d u$ sentimentalisme. Cette remise en cause du modèle de la fiction sentimentaliste ne laissait aux prosateurs du début $\mathrm{du} \mathrm{xIX}^{\mathrm{e}}$ siècle que deux voies possibles. Soit l'évitement, passant par la redécouverte et le développement des modèles proposés au XVIII ${ }^{\mathrm{e}}$ siècle par la prose pré-karamzinienne, soit la revitalisation du modèle sentimentaliste via sa diversification ou son autocritique. C'est à la première voie qu'est consacrée la troisième section du livre d'A. Tosi.

4 Dans cette partie, l'auteur présente, à travers l'analyse de cinq œuvres spécifiques, les différents genres réactivés pour servir d'alternative au récit karamzinien. Il s'agit d'abord du récit oriental (vostočnaja povest'), présenté comme la variante russe du conte philosophique, et analysé à travers l'exemple du récit Na drugoj den' (Le lendemain, 1809) d'Aleksandr Benickij. D'inspiration voltairienne, ce genre permit l'apparition d'un narrateur objectif, opposé au narrateur compatissant du sentimentalisme, et l'épurement du style par rejet des poétismes karamziniens. Un autre genre est le récit mondain (svetskaja povest'), illustré par Laure, œuvre en français publiée en 1819 par Zinaida Volkonskaja. Puisant dans le réalisme psychologique $d u X \mathrm{XIII}^{\mathrm{e}}$ siècle, ce récit oppose à la représentation sentimentaliste standardisée de la femme bonne épouse et bonne mère, une femme aliénée par ces deux états, et brosse un tableau de la bonne société d'une ironie sévère, annonçant Griboedov plus qu'il ne prolonge Karamzin. Le recueil Črezvyčajnye proisšestvija (Événements extraordinaires, 1809) d'Andrej Kropotov illustre une troisième alternative au sentimentalisme. Prolongeant la tradition picaresque du $\mathrm{XVIII}^{\mathrm{e}}$ siècle en l'enrichissant de jeux métatextuels sterniens, cette œuvre délaisse le ton sérieux des épigones de Karamzin, en allant parfois jusqu'au grotesque. Identifié par A. Tosi comme une imitation d'un pamphlet de Sterne, Istorija o smurom kaftane (L'histoire du caftan brun), un des récits composant le recueil,apparaît ainsi comme une source possible du Manteau de Gogol'. Les deux derniers types de fiction opposés à la norme sentimentaliste, enfin, sont illustrés par le roman de mœurs d'Aleksandr Izmailov Evgenij, ili pagubnye sledstvija durnogo vospitanija i soobščestva (Eugène ou les conséquences désastreuses d'une mauvaise éducation et de mauvaises fréquentations, 1799-1801), et le récit de voyage de Savelij Fon Ferel'ts Putešestvie kritiki (Le Voyage d'un critique, 1818). Le premier introduit dans la prose fictionnelle le thème, emprunté à la comédie de mœurs et au journalisme du XVIII ${ }^{\mathrm{e}}$ siècle, de la gallomanie, tandis que le second reprend en la prolongeant la critique radicale du servage inaugurée par Radiščev, en l'intégrant au modèle du récit épistolaire hérité des Lettres d'un voyageur russe.

5 À ces différentes tentatives pour contourner les codes du sentimentalisme s'opposèrent les efforts de divers auteurs pour les renouveler. Détaillés dans la quatrième partie, ces efforts montrent à quel point il est inexact de penser que le sentimentalisme aurait épuisé ses possibilités avec Karamzin. Rompant avec la représentation traditionnelle de la femme, illustrée entre autres par le récit de Marija Izvezkova Émilie (Emilija, 1806), le récit anonyme Russkaja amazonka (L'Amazone russe, 1809) en présente ainsi une vision 
renouvelée, où la femme, dans certaines circonstances historiques bien spécifiques, gagne la liberté de devenir maitresse de son sort. Un autre moyen pour renouveler le sentimentalisme consista à recourir à l'auto-ironie, en suivant l'exemple même suggéré par la double dimension de l'œuvre de Sterne. C'est ce que fit Nikolaj Brusilov dans son court roman Bednyj Leandr (Le Pauvre Léandre, 1803), qui écorne le rapport superficiel à la littérature du monde des salons. Mis à la mode par Les Lettres d'un voyageur russe, le récit de voyage sentimental connut lui aussi un renouvellement de l'intérieur, par le recours à la parodie dans le cas de Moe putešestvie ili priključenie odnogo dnja (Mon voyage ou aventure d'un jour, 1803) du même Brusilov et du Čuvstvitel'noe putešestvie po Nevskomu prospektu (Voyage sentimental le long de la perspective Nevski, 1820) de Pavel Jakovlev, ou par une révision de l'équilibre entre sensibilité et description documentaire - au profit de cette dernière - dans le Putešestvie po Saksonii, Avstrii i Italii (Voyage en Saxe, Autriche et Italie, 1805) de Fedor Lubjanovskij.

6 La cinquième et dernière partie de l'ouvrage d'A. Tosi, enfin, porte sur la concurrence imposée à ces deux voies (anti-sentimentaliste et sentimentaliste renouvelée) par celle du préromantisme, importé d'Europe occidentale et marqué par son goût pour le passé. S'il apparait dès le XviII siècle en Russie, l'intérêt pour le passé et l'histoire n'y est encore qu'historicisme et ne peut pas être traduit en littérature par la langue du sentimentalisme. Ce n'est ainsi que sous Alexandre qu'il s'épanouit vraiment. Dans le champ de la prose de fiction, cet intérêt nouveau emprunte diverses formes, analysées par l'auteur. À côté du travail peu convaincant d'épigones de Karamzin comme Pëtr Kazotti, dont la vision du passé est prisonnière de la poétique de l'idylle, tranche celui de deux historiens écrivains à leurs heures, Nikolaj Arcybyšev et Gavril Gerakov, dont les connaissances scientifiques renouvelèrent le matériau littéraire. Un autre progrès est introduit par les récits inspirés de l'histoire récente des guerres napoléoniennes. Nevidimka, ili tainstvennaja ženččina (L'Invisible ou la femme mystérieuse, 1815), et L'Amazone russe, évoqué plus haut, surent donner à l'histoire un rôle dynamique dans leur intrigue. Enfin, l'intérêt pour l'histoire à l'époque d'Alexandre apparaît dans la mode du roman gothique, qui inspira Gnedič mais également Žukovskij. Plus connu pour sa poésie, notamment sa traduction de L''liade, le premier écrivit un roman historique, Don Korrado, auquel A. Tosi rend son importance historique et esthétique. Illustration du passage du récit de terreur au récit d'horreur, ce texte emprunte à Schiller et Milton leur fascination pour le surhomme démoniaque. Žukovskij, quant à lui, se révèle un parfait élève du roman gothique dans son récit Mar'ina rošča (Le Bois de Maria), mais aussi dans sa célèbre ballade Ljudmila. Comme le conclut l'auteur, le recours à la description de l'angoisse et de la peur éprouvées par les héros des fictions gothiques permit aux préromantiques de complexifier la psychologie simpliste des personnages sentimentalistes.

7 Alternant études précises d'œuvres souvent injustement méconnues et généralisations historiques, l'ouvrage fourmille d'idées originales, notamment grâce à son recours aux gender studies, sans oublier cependant de remplir son rôle de synthèse historique. D'une grande érudition, ainsi qu'en attestent les références nombreuses à la critique des années 1800-1810, Waiting for Pushkin est également remarquable par sa capacité à questionner les hiérarchies de l'histoire littéraire moderne, qui juge souvent hâtivement comme secondaires des textes pourtant intéressants. L'étude thématique et narratologique des œuvres menée par A. Tosi n'oublie jamais enfin de s'enrichir de remarques linguistiques, afin de montrer le lent processus menant au dépassement des limites thématico- 
génériques imposées à la littérature russe du début du XIX siècle par la langue trop élégante de Karamzin.

8 Aussi ne pourra-t-on que regretter, au regard de toutes ces qualités, que l'ouvrage trahisse parfois un certain flou dans l'emploi des termes. Ainsi il semble difficile de qualifier Les Confessions de « sentimental novel of travel» (p. 268). Le livre de Rousseau n'est en effet ni un roman, ni un récit de voyage. De même, il parait hasardeux d'introduire une équivalence parfaite entre conte philosophique et « vostočnaja povest'» (p. 111). Tous les contes philosophiques ne furent pas orientaux. En attestent Micromégas ou L'Ingénu ! On regrettera surtout que le recours au terme trop large de "fiction", regroupant indistinctement récit, nouvelle et roman, empêche A. Tosi d'étudier les relations intragénériques, notamment de hiérarchie, reliant ces diverses formes. On relève également quelques inexactitudes. Une scène de viol du Voyage d'un critique est ainsi présentée comme la première scène aussi crue de la littérature russe (p. 182). C'est oublier les scènes similaires du Voyage de Saint-Pétersbourg à Moscou, dont l'auteur souligne pourtant l'influence sur Fon Ferel'ts. "The policeman of Europe », ensuite, ne fut pas Alexandre $\mathrm{I}^{\text {er }}$ (p. 33), mais son frère Nicolas. Quant à Catherine II, elle ne succéda pas à Pierre II (p. 106), mais à Pierre III. Enfin, l'auteur du Voyage autour de ma chambre n'est pas François-Xavier de Maistre (p. 247), sénateur au parlement de Chambéry, mais son fils Xavier. En outre, on déplorera un grand nombre d'inexactitudes dans la transcription et parfois même la traduction du russe, et des fautes d'orthographe dans les mots français, ainsi que dans les titres russes de la bibliographie finale. Ces négligences sont d'autant plus regrettables que le livre d'A. Tosi, par l'originalité de son objet et l'intérêt de ses conclusions, est amené à devenir un ouvrage de référence. Souhaitons qu'une future réédition en débarrasse le texte, par ailleurs tout à fait passionnant. 